\title{
Cooperation in the Prisoner's Dilemma game in Random Scale-Free Graphs
}

\author{
Julia Poncela \\ Institute for Biocomputation and Physics of Complex Systems (BIFI), University of Zaragoza, Zaragoza E-50009, Spain \\ Jesús Gómez-Gardeñeł \\ Institute for Biocomputation and Physics of Complex Systems (BIFI), University of Zaragoza, Zaragoza E-50009, Spain \\ Departament d'Enginyeria Informática i Matemátiques, \\ University Rovira i Virgili, Tarragona E-43007, Spain and \\ Scuola Superiore di Catania, University of Catania, Catania I-95123, Italy \\ Yamir Moreno \\ Institute for Biocomputation and Physics of Complex Systems (BIFI), \\ University of Zaragoza, Zaragoza E-50009, Spain and \\ Department of Theoretical Physics, University of Zaragoza, Zaragoza E-50009, Spain \\ Luis Mario Floría \\ Institute for Biocomputation and Physics of Complex Systems (BIFI), \\ University of Zaragoza, Zaragoza E-50009, Spain and \\ Department of Condensed Matter Physics, University of Zaragoza, Zaragoza E-50009, Spain
}

(Dated: October 26, 2018)

\begin{abstract}
In this paper we study the cooperative behavior of agents playing the Prisoner's Dilemma game in random scale-free networks. We show that the survival of cooperation is enhanced with respect to random homogeneous graphs but, on the other hand, decreases when compared to that found in Barabási-Albert scale-free networks. We show that the latter decrease is related with the structure of cooperation. Additionally, we present a mean field approximation for studying evolutionary dynamics in networks with no degree-degree correlations and with arbitrary degree distribution. The mean field approach is similar to the one used for describing the disease spreading in complex networks, making a further compartmentalization of the strategists partition into degreeclasses. We show that this kind of approximation is suitable to describe the behavior of the system for a particular set of initial conditions, such as the placement of cooperators in the higher-degree classes, while it fails to reproduce the level of cooperation observed in the numerical simulations for arbitrary initial configurations.
\end{abstract}

PACS numbers: 87.23.Kg, 02.50.Le, 89.75.Fb

\section{INTRODUCTION}

The ubiquity of cooperation at all scales of life's organization from genes, through multicellular organisms, to animal and human societies, has not been immediately accommodated in the theory of evolution [Nowak, 2006]. In fact, to understand the observed survival of cooperative behavior when selfish actions provide a higher reproductive success (fitness) several mechanisms (non mutually exclusive) have been proposed: a) Kin selection, based on genetic relatedness, b) group selection, in which the demes (groups) instead of organisms are the entities on which natural selection acts upon, and c) reciprocal altruism, which includes direct reciprocity (through repeated interactions) and diverse types of indirect reciprocity (through reputation, scoring, punishment, signaling, etc...).

Perhaps the simplest (i.e. less demanding) mechanism, termed lattice (or network) reciprocity, consists of assuming that each agent interacts only with its neighbors as given by a network of social contacts. Early pioneering numerical work by Nowak and May [Nowak \& May, 1992] on the evolutionary

\footnotetext{
*To whom correspondence should be addressed. E-mail: gar-
} denes@gmail.com dynamics of the Prisoner's Dilemma game (see below) in twodimensional regular (square) lattices, showed that the cooperative phenotype was not driven to extinction. This result stimulated much work paying attention to the evolution of cooperation in graph-structured populations [Szabó \& Fáth, 2007]. It has been well recognized that graph topologies play a crucial role in providing positive feedback evolutionary mechanisms that facilitate the asymptotic survival of cooperation. Therefore, it is necessary to change the benchmark of evolutionary game theory from unstructured or "well-mixed populations to the more realistic case of complex networks.

Interactions in animal and human social systems are, in general, modelled on sets of individuals playing diverse social dilemmas games. In these games, players can adopt two possible strategies: cooperation (C) and defection (D). In the classical setting, a player $i$ plays with all the other individuals in the system accumulating, in each of these games, a payoff that depends on both its strategy and the one adopted by its corresponding opponent. In particular, both $\mathrm{C}$ and $\mathrm{D}$ receive $R$ (reward) under mutual cooperation and $P$ (punishment) under mutual defection, while $\mathrm{C}$ receives $S$ (suckers) when confronted to $\mathrm{D}$, which in turn receives $T$ (temptation). The specific values that take the latter payoffs define the specific social dilemma we are dealing with. From now on, we will consider the case $T>R>P>S$ so that we will focus 
on the Prisoner's Dilemma game [Hamilton, 1964; Axelrod \& Hamilton, 1981; Hofbauer \& Sigmund, 1998; Hofbauer \& Sigmund, 2003; Nowak \& Sigmund, 2005].

Once every agent has played the game with the rest of the population, i.e. has completed a game's round-robin, selection takes place and each player is allowed to change its strategy. Following the replicator dynamics [Hofbauer \& Sigmund 1998, Gintis 2000], the probability that an agent adopt a different strategy in the next generation depends on the difference between its payoff and the average payoff of the system. Under these conditions (well-mixed hypothesis or mean field approximation), the fraction of cooperators will unavoidably decrease in time towards zero. Therefore, it seems clear that in order to answer the question about how cooperative behavior can survive in animal and human social systems one has to relax the hypothesis considered above, such as the well-mixing assumption.

In the last decade, scientist have unveiled the structure of many complex systems, and have described them in terms of complex networks [Bornholdt \& Schuster, 2003; Dorogovtsev \& Mendes, 2003; Newman, 2003; Boccaletti, Latora, Moreno, Chavez \& Hwang, 2006]. These networks are the backbone of complex systems and hence they are the substrates on top of which a number of relevant dynamics (such as disease spreading, information transmission or human traits) occur. The structure of complex networks is far from being described with a fixed value $k$ accounting for the typical number of interactions that an element shares with the rest of the system. On the other hand, it has been measured that the distribution of the number of contacts (or alternatively the degree) of the elements follows a power-law, $P(k) \sim k^{-\gamma}$, i.e. the networks are scale-free (SF). Moreover, many measures of real complex networks have obtained an exponent $2<\gamma<3$ for the powerlaw degree distribution, pointing out that the variance $\left\langle k^{2}\right\rangle$ of this statistical distribution diverges in the infinite population limit. Taking into account these results, it becomes clear that any mathematical model or numerical simulation aimed at describing the cooperative behavior in real social systems has to incorporate the scale-free nature of the social contact network.

In this paper we will study the cooperation in random scalefree networks. First, in section II, we will analyze the evolution and the structure of cooperation in the prisoner's dilemma game through numerical simulations of the evolutionary dynamics. In particular, we will study the average level of cooperation sustained by random SF networks and how cooperators and defectors are arranged across the network. We will compare our results with those obtained by previous studies on other, scale-free or not, network topologies. In the second part of the paper, section III, we present a mean field approximation that divides players into classes accordingly to their number of contacts. The mean field approximation is shown to be correct for particular sets of initial conditions and hence provides a useful analytical tool for studying the cooperation in complex networks (in particular for those graphs with a long-tailed degree distribution). Finally, in section IV we round off the paper by summarizing the main results of the work.

\section{THE STRUCTURE OF COOPERATION IN RANDOM SCALE-FREE NETWORKS}

In the recent years, a number of studies have studied the prisoner's dilemma on top of complex networks [Eguíluz, Zimmermann, Cela-Conde \& San Miguel, 2005; Lieberman, Hauert \& Nowak, 2005; Santos \& Pacheco, 2005; Ohtsuki, Hauert, Lieberman \& Nowak, 2006; Santos \& Pacheco, 2006; Santos, Pacheco \& Lenaerts, 2006; Gómez-Gardeñes, Campillo, Floría \& Moreno, 2007; Poncela, Gómez-Gardeñes, Floría \& Moreno, 2007; Szabó \& Fath, 2007; Gómez-Gardeñes, Poncela, Floría \& Moreno, 2008; Szolnoki, Perc \& Danku, 2008; Vukov, Szabó \& Szolnoki, 2008]. These works have determined that SF networks enhance cooperation when compared to homogeneous random networks such as Erdös-Rényi graphs (described by a Poisson degree distribution, $P(k) \sim \mathrm{e}^{\langle k\rangle}\langle k\rangle^{k} / k$ !). However, these works have mainly focused on SF networks constructed via the celebrated Barabasi-Albert (BA) model [Barabási \& Albert, 1999]. The BA model considers that the network is grown from an initial core of $m_{0}$ nodes, incorporating a new node to the network every time step. Every new node launches $m$ links to the nodes already present in the growing network following a preferential attachment rule (i.e. the probability of receiving a link from the new node is proportional to the degree of the nodes).

The networks generated using the BA model have a powerlaw degree distribution with $\gamma=3$ but, at the same time, they posses important features that make them different from random SF networks constructed by means of purely statistical algorithms such as the Molloy-Reed configurational model [Molloy \& Reed, 1998]. These differences are manifested in the so-called age-correlations [Dorogovtsev \& Mendes, 2003; Newman, 2003] that have as a consequence the interconnection of highly-connected elements or hubs. The links between hubs have been shown to play a crucial role in the survival of cooperation [Santos \& Pacheco, 2005] since when they are removed the cooperation level decreases notably (although it remains larger than those observed in regular and homogeneous graphs).

In fact, a careful inspection of the structure of cooperation in BA SF networks [Gómez-Gardeñes, Campillo, Floría \& Moreno, 2007] reveals that cooperators are arranged in a very cohesive way. In particular, they are glued together into a single cooperator cluster sustained by the highly connected nodes that always play as cooperators [Gómez-Gardeñes, Poncela, Floría \& Moreno, 2008]. Our first goal in the study of random SF networks is to check if the deletion of the hub-to-hub links affects the structure of cooperation observed in BA networks, explaining qualitatively the drop in the cooperation level as a break down of the cohesive arrangement of cooperators.

To study the structure of cooperation in random SF networks we have performed a rewiring of the SF networks obtained by means of the BA model. Following the scheme shown in [Maslov \& Sneppen, 2002] the rewiring process destroys any kind of correlations present in the original network preserving the degree sequence of the graph, and thus keeping the same degree distribution $\left(P(k) \sim k^{-3}\right)$ as in the origi- 
nal BA network. The networks generated in this work have $N=4000$ and $\langle k\rangle=4$.

Once the network is constructed, we perform the numerical simulation of the evolutionary dynamics as dictated by the prisoner's dilemma payoff matrix with $P=S=0, R=1$, $T=b>1$, so that we deal with only one control parameter: the temptation to defect $b$. We start from an initial configuration with equal number of $C$ and $D$ players $(c=d=N / 2)$ that are randomly distributed across the network nodes. At each generation of the discrete evolutionary time $t$, each agent $i$ plays once with every agent in its neighborhood and accumulates the obtained payoffs $P_{i}$. Then, all the players update synchronously their strategies by the following rules. Each individual $i$ chooses at random a neighbor $j$ and compares its payoff $P_{i}$ with $P_{j}$. If $P_{i}>P j$, player $i$ keeps the same strategy for the next generation. On the other hand, if $P_{j}>P_{i}$, the player $i$ adopts the strategy of its neighbor $j$ for the next game round robin with probability [Santos \& Pacheco, 2005]:

$$
\Pi_{i \rightarrow j}=\beta\left(P_{j}-P_{i}\right) .
$$

Here, $\beta$ is the characteristic inverse time scale: the larger $\beta$ the faster evolution takes place. Additionally, we consider $\beta=$ $\left(\max \left\{k_{i}, k_{j}\right\} b\right)^{-1}$ assuring that $\Pi_{i \rightarrow j} \leq 1$.

Let us now explain the details of the numerical analysis. We let the system evolve until a stationary regime is reached. The stationary regime is characterized by a stable average level of cooperation $\langle c\rangle$, that is the fraction of $\mathrm{C}$ players in the network, $\langle c\rangle=c / N$. To compute $\langle c\rangle$ we let the dynamics evolve over a transient time $\tau_{0}=5 \cdot 10^{3}$, and we further evolve the system over time windows of $\tau=10^{3}$ generations. In each time window, we compute the average value and the fluctuations of $c(t)$. When the fluctuations are less than or equal to $1 / \sqrt{N}$, we stop the simulation and consider the average cooperation obtained in the last time window as the asymptotic average cooperation $\langle c\rangle$. In order to make an extensive sampling of initial conditions and network realizations we have performed $10^{3}$ numerical simulations for each value of the temptation $b$ studied, and averaged accordingly the values $\langle c\rangle$ found in the realizations. In figure 1 a we have plotted the evolution of the average level of cooperation $\langle c\rangle$ as a function of $b$. Our results confirm the findings of [Santos \& Pacheco, 2005]: the removal of age-correlations makes SF networks less robust to defection than BA networks. However, the figure shows that $\langle c\rangle(b)>0$ until larger values of $b$ as compared to the cooperation levels found for homogeneous ER graphs [Gómez-Gardeñes, Campillo, Floría \& Moreno, 2007].

To measure the structure of cooperation we follow the approach introduced in [Gómez-Gardeñes, Campillo, Floría \& Moreno, 2007]. Once the system has reached the stationary regime, we let the system evolve again for $\tau_{s}=10^{4}$ additional time steps, and measure the relevant magnitudes for the dynamical characterization of the stationary state. In particular, we label each player in one of the following three categories: pure cooperators $(\mathrm{PC})$, pure defectors $(\mathrm{PD})$ and fluctuating (F) players. Pure cooperator (pure defector) strategists are those players that adopt cooperation (defection) during all the $\tau_{s}$ generations. Conversely, fluctuating players are those agents that play both as defectors and cooperators at the sta-
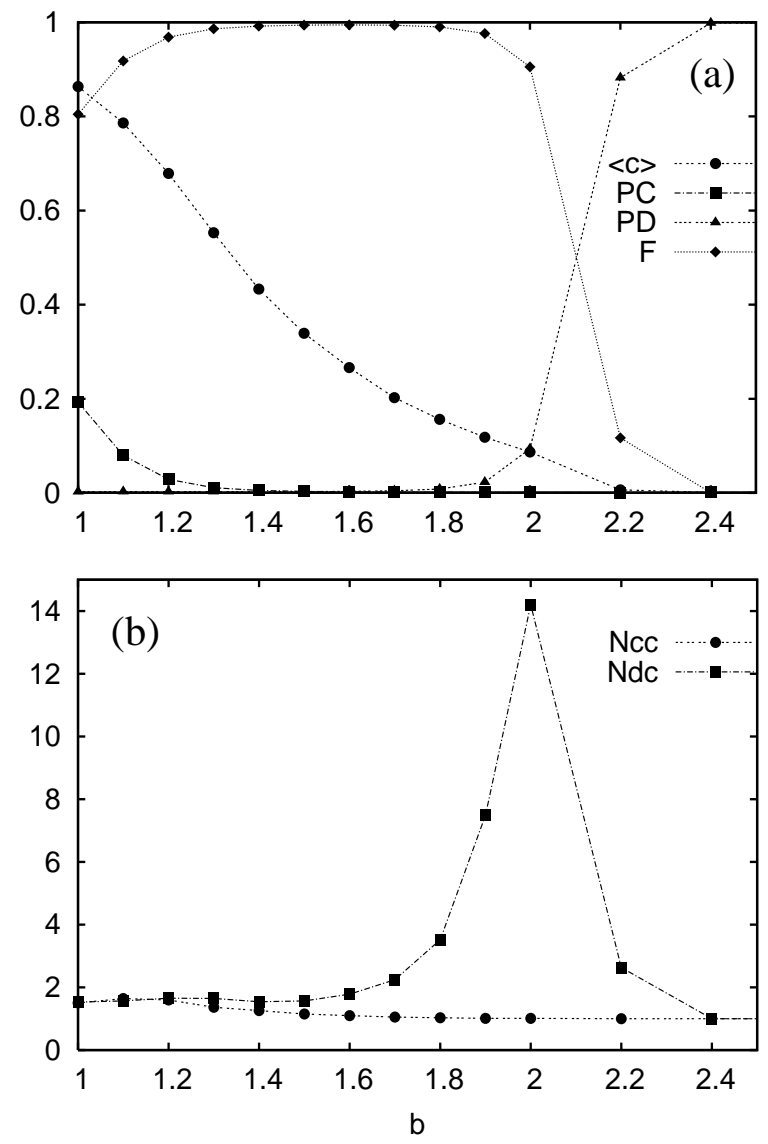

FIG. 1: (a) Evolution of the average level of cooperation $\langle c\rangle$ as a function of the temptation to defect $b$ in random SF graphs. The panel also shows the corresponding evolution for the fraction of pure cooperators (PC), pure defectors (PD) and fluctuating (F) players. (b) Evolution of the number of cooperator clusters $N_{c c}$ and defector clusters $N_{d c}$ as a function of $b$.

tionary regime, and hence change their strategies during the time window of length $\tau_{s}$. In figure 1 a we also plot the fraction of $\mathrm{PC}, \mathrm{PD}$, and $\mathrm{F}$ players as a function of the temptation $b$. The evolution of the three fractions follows the expected behavior: PC decrease with $b$, whereas F players first increase and occupy macroscopically the network. Finally, F agents are progressively replaced by PD until all the network is fully occupied by pure defectors. Remarkably, the number of PC is surprisingly lower than both BA and homogeneous ER networks. Instead, in random SF graphs the average level of cooperation is sustained by those $\mathrm{F}$ players that dominate the population of the network in the range $1.2<b<1.9$.

Once we have identified the PC, PD and F players we define the cooperator and defector clusters. A cooperator (defector) cluster is a connected subgraph composed of nodes that are pure cooperators (defectors) and the links between them. In figure 1 $\mathrm{b}$ we have plotted the number of cooperators and defectors clusters as a function of $b$. The first difference with respect to BA networks is that here we find realizations with more than one cooperator cluster. This difference explains the 
drop in the cooperation level observed in [Santos \& Pacheco, 2005]: the more fragmented the cooperators are arranged the less sources of benefits they find in their surroundings and the larger is the probability to be invaded by the instantaneous defectors that are in contact with them. Regarding the defector clusters we observe the same picture as in BA networks: PD are arranged in several clusters when they start to invade the network $(b \simeq 2)$. The number of defector clusters decrease as they start to grow in size and finally collapse into a single one only when all the network is totally invaded by pure defectors. We have also checked the probability that a node of degree $k$ is a cooperator in the stationary regime. Our numerical simulations show that, in agreement with previous numerical observations in BA networks [Gómez-Gardeñes, Poncela, Floría $\&$ Moreno, 2008], high degree nodes are more likely to act as cooperators than intermediate or low degree individuals.

Summing up the previous results, in random SF networks, the fragmentation of the cooperator clusters together with the extremely low fraction of pure cooperators and the prevalence of fluctuating individuals lead to an organization of cooperation that is radically different to that observed in BA SF networks.

\section{THE DEGREE-BASED MEAN FIELD APPROXIMATION}

The random SF graphs analyzed above are free of any kind of correlation between the properties (age, degree, etc...) of two adjacent nodes. Therefore, it is amenable to study analytically the evolution of the cooperation by considering a similar approach to that used for diffusion processes in complex networks [Pastor-Satorras \& Vespignani, 2001a; Pastor-Satorras \& Vespignani, 2001b; Moreno, Pastor-Satorras \& Vespignani, 2002] with arbitrary degree distributions and no correlations. To incorporate the heterogeneity in the number of social contacts of individuals we make a further compartmentalization of the strategists in degree-classes. In this sense, we label $c_{k}$ and $d_{k}$ as the fraction of cooperators and defectors with degree $k$ so that the total number of cooperators and defectors is, respectively

$$
\begin{aligned}
& c=N \sum_{k} P(k) c_{k}, \\
& d=N \sum_{k} P(k) d_{k} .
\end{aligned}
$$

Obviously the relation $c_{k}+d_{k}=1$ holds and therefore we write the evolution of the fraction of cooperators with degree $k$ as

$$
\dot{c_{k}}=\left(1-c_{k}\right) \Pi_{k}^{D C}-c_{k} \Pi_{k}^{C D}
$$

where $\Pi_{k}^{D C}\left(\Pi_{k}^{C D}\right)$ is the probability that a cooperator (defector) of degree $k$ change its strategy to defection (cooperation). Assuming that the network has no degree-degree correlations, and following the replicator-like update rule [1], we can write the probabilities $\Pi_{k}^{D C}$ and $\Pi_{k}^{C D}$ as

$$
\begin{aligned}
\Pi_{k}^{D C} & =\sum_{k^{\prime}} \frac{k^{\prime} P\left(k^{\prime}\right)}{\langle k\rangle} \beta \Theta\left[P_{k^{\prime}}^{C}-P_{k}^{D}\right] c_{k^{\prime}}, \\
\Pi_{k}^{C D} & =\sum_{k^{\prime}} \frac{k^{\prime} P\left(k^{\prime}\right)}{\langle k\rangle} \beta \Theta\left[P_{k^{\prime}}^{D}-P_{k}^{C}\right]\left(1-c_{k^{\prime}}\right),
\end{aligned}
$$

where the function $\Theta[x]$ is defined as $\Theta[x]=x$ if $x>0$ and $\Theta[x]=0$ otherwise. Besides, $P_{k}^{C}$ and $P_{k}^{D}$ are the payoffs obtained by a cooperator and a defector of degree $k$ respectively, and can be written as

$$
\begin{aligned}
P_{k}^{C} & =k \sum_{k^{\prime}} \frac{k^{\prime} P\left(k^{\prime}\right)}{\langle k\rangle} c_{k^{\prime}}=k l_{c}, \\
P_{k}^{D} & =b \cdot k l_{c},
\end{aligned}
$$

where $l_{c}$ is the probability that a node has a cooperator neighbor. Now we can insert the above two expressions (7) and (8) in equations (6) and (5) and finally write the evolution equation (4) as

$$
\begin{aligned}
\dot{c_{k}} & =\left(1-c_{k}\right) \sum_{k^{\prime}>b k} \frac{k^{\prime} P\left(k^{\prime}\right)}{\langle k\rangle} \beta l_{c}\left(k^{\prime}-b k\right) c_{k^{\prime}} \\
& -c_{k} \sum_{k^{\prime}>b k} \frac{k^{\prime} P\left(k^{\prime}\right)}{\langle k\rangle} \beta l_{c}\left(b k^{\prime}-k\right)\left(1-c_{k^{\prime}}\right) \\
& -c_{k} \sum_{k^{\prime}>k / b}^{b k} \frac{k^{\prime} P\left(k^{\prime}\right)}{\langle k\rangle} \beta l_{c}\left(b k^{\prime}-k\right)\left(1-c_{k^{\prime}}\right),
\end{aligned}
$$

where we have separated the contributions to the transition $\mathrm{C} \rightarrow \mathrm{D}$ that come from neighbors with $k^{\prime}>b k$ and $k^{\prime}<b k$, so that it is clear that the number of degree classes that participate in the transition $\mathrm{C} \rightarrow \mathrm{D}$ is larger than those that influence the change $\mathrm{D} \rightarrow \mathrm{C}$.

The main assumption behind the above mean field approach is that the average level of cooperation inside a degree-class, $c_{k}$, is a proper magnitude for describing the state of nodes with this degree. In particular, this assumption is strictly correct when $c_{k}$ is either 1 or 0 . This motivate us to study the solution of equations (9) using a particular set of initial conditions: the targeted cooperation.

We define targeted cooperation as a set of initial conditions for of the system (9) for which $c_{k}(0)=1$ if $k>k^{*}$ and $c_{k}(0)=0$ if $k<k^{*}$. We have carefully explored the solutions of equations 9 when $P(k)$ is a power-law degree distribution. To this end, we have studied power-laws with several values of $\gamma$ and used different values for the degree threshold $k^{*}$. The numerical solution of equations (9) reveals that the cooperation level remains for $b>1$, reaching a stationary value that depends on both the value of $b$ and that of the threshold $k^{*}$. In figure 2 we show the time evolution of the average level of cooperation for several values of $b$ and $k^{*}=2$ and $k^{*}=3$. The degree distribution in the figure is a powerlaw with $\gamma=3$. The solutions show that the larger $k^{*}$ and/or $b$ are the lower the cooperation level is. 

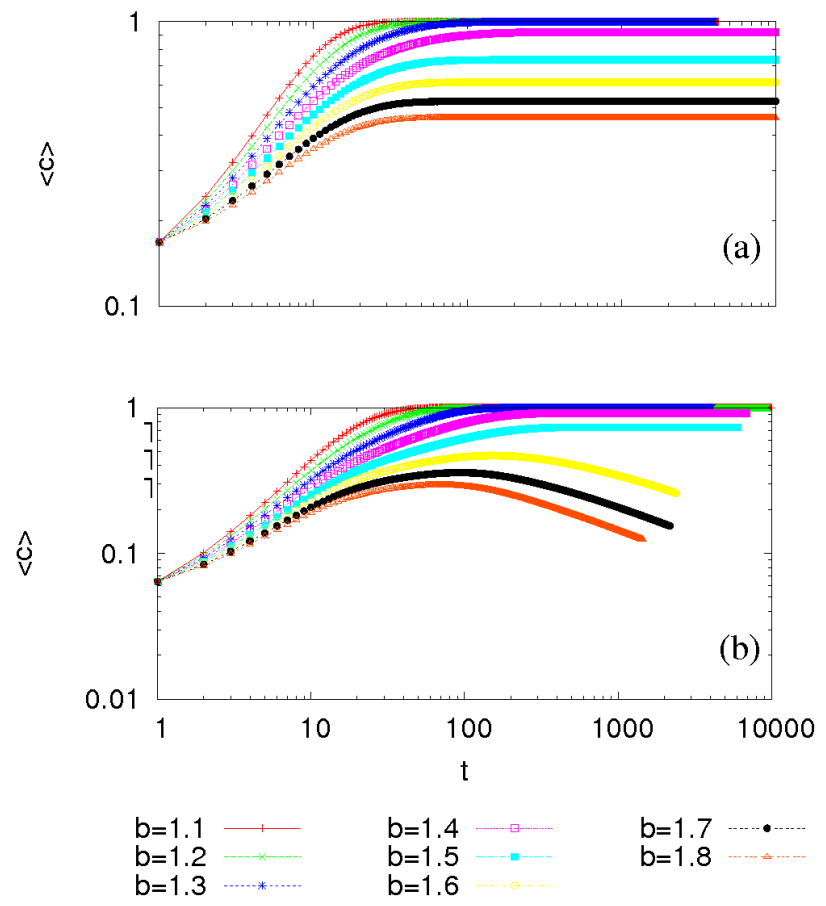

FIG. 2: (Color online). The two panels show the time evolution of the fraction of cooperators $\langle c\rangle(t)$ obtained solving equation (9) when targeted cooperation is used as initial conditions and being $P(k)$ a power-law with $\gamma=3$. The different curves correspond to several values of $b$ (as shown in the bottom of the figure). The targeted cooperation used correspond to (a) $k^{*}=2$ and (b) $k^{*}=3$.

It is interesting to study in detail the effect of the threshold $k^{*}$ over the asymptotic level of cooperation. In particular, we can obtain the minimum amount of degree classes that we have to fill initially with cooperators so that cooperation is able to survive asymptotically in the network. We have explored different sets of initial conditions corresponding to different values of $k^{*}$. Starting from a low value of $k^{*}$ we have solved equations (9) and computed the final level of cooperation $\langle c\rangle$. If $\langle c\rangle>0$ we increase the value of $k^{*}$ and solve again the system (9). This process is iterated until we reach a value $k_{c}^{*}$ for which cooperation vanishes. The computed value $k_{c}^{*}$ thus represents the minimal amount of cooperator degree classes needed at time 0 to sustain asymptotically a nonzero level of cooperation. In figure 3 we have plotted the functions $k_{c}^{*}(b)$ for three power-law degree distributions $(\gamma=2$, $3,4)$. Obviously, we observe that as the temptation to defect increases it is necessary to fill more degree classes to assure a nonzero level of cooperation. More interestingly, we show that the heterogeneity of the network increases the value of $k_{c}^{*}$. This results is related to the fact that filling a given amount of degree classes is more efficient (more nodes are initially set as cooperators) when the network is more heterogeneous.

Computing the phase diagram $k_{c}^{*}(b)$ is a difficult task if we only rely on the results of the numerical simulations of the evolutionary dynamics on top of the graphs. Therefore, the

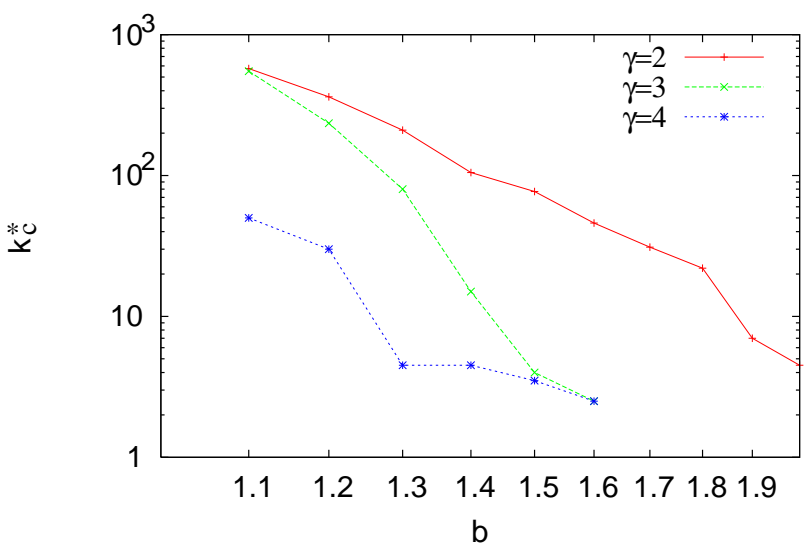

FIG. 3: Phase diagram $k_{c}^{*}(b)$. The three curves correspond to different power-law distributions (namely, $\gamma=4,3$ and 2). Each curve $k_{c}^{*}(b)$ represent the border between two different asymptotic regimes for the evolution of equations 9 with targeted cooperation: The area below the curves correspond to the points $\left(b, k^{*}\right)$ where targeted cooperation yield nonzero asymptotic level of cooperation. Conversely, the area above the curves correspond to the targeted initial conditions for which the evolution of equations 9 yields $\langle c\rangle \rightarrow 0$.

mean field approach represents, in this context, a useful tool for substituting computationally expensive numerical simulations. However, how accurate are the results of the solutions of equations (9) when compared to numerical simulations? To check the reliability of the degree-based mean field approach in the context of targeted cooperation we have computed the diagram $\langle c\rangle(b)$ for random SF networks with $\gamma=3$ using two different sets of initial conditions corresponding to $k^{*}=3$ and 4. In figure 4 we show the results of the numerical simulations compared to the results obtained by solving equations (9). Obviously, the agreement is not complete but the evolution of the cooperation as a function of $b$ follows the same qualitative behavior and the cooperation tends to zero $(\langle c\rangle \gtrsim 0)$ around the same values of $b$. The values of $b$ for which $\langle c\rangle=0$ in each of the curves of the figures are obviously related to the values $k_{c}^{*}$. Our results show that the curves $\langle c\rangle(b)$ obtained from numerical simulations reach larger values of $b$ with $\langle c\rangle>0$. On the other hand, the numerical simulations yield very low values of $\langle c\rangle$ for those values of $b$ for which cooperation asymptotically vanishes solving equations (9). Therefore, the mean field approach seems to be of help to study the behavior of $k_{c}^{*}(b)$ and the asymptotic level of cooperation of the system when targeted cooperation is initially placed in the system.

Regarding general (i.e. non-targeted cooperation type of) initial conditions for the degree-based mean field equations (9), some comments are in order. For both, power-law and Poisson degree distributions $P(k)$, random uniformly distributed values for $c_{k}(0)$, as well as fixed value $c_{k}(0)=0.5$ (mimicking the initial conditions in the numerical simulations of previous section), led to asymptotic zero level of cooperation as soon as $b>1$. This suggests that, generically speaking, mean field approaches (even in generalized forms, as equations (9)) to the evolutionary dynamics of prisoner's 


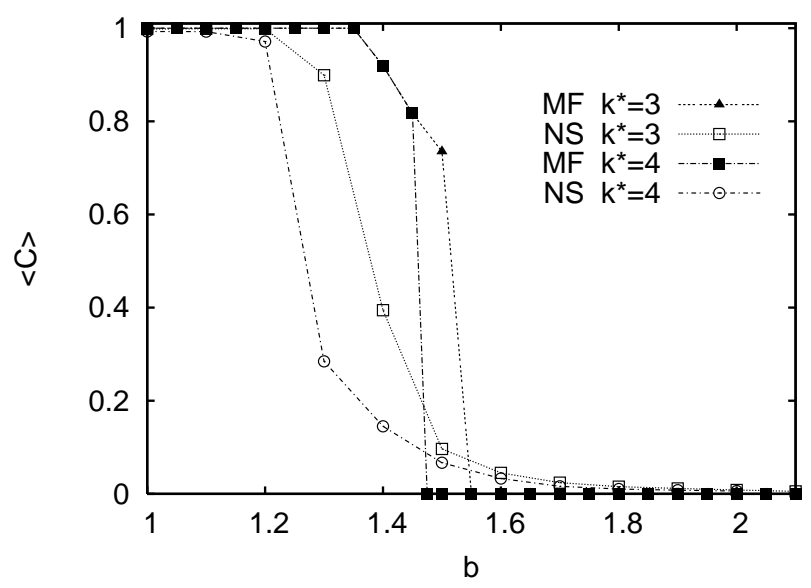

FIG. 4: Evolution of the asymptotic level of cooperation $>$ obtained when $(i)$ solving the mean field (MF) equations 9 and (ii) computed through numerical simulations (NS) of the evolutionary dynamics on top of a random SF network. The degree distribution used is a powerlaw with $\gamma=3$. In both cases we have set targeted cooperation as initial conditions for the evolutionary dynamics. We have used $k^{*}=3$ and 4 .

dilemma games on graphs are likely bound to fail to account for the numerically observed survival of cooperation. This would be in agreement with results reported elsewhere [Floría, Gracia-Lázaro, Gómez-Gardeñes \& Moreno, 2008] on a particular type of artificial networks that allow a rigorous analysis of the issue. To put it in plain terms, the lattice reciprocity mechanisms that enhance the evolutionary survival of cooperation in network settings seem to be out of reach from the (homogeneity) mean field assumptions, in the sense that they are associated in an essential way to fluctuations of averaged quantities, like $c_{k}$ which are the basic descriptors in mean field approaches.

\section{CONCLUSIONS}

Scale-free networks have been recently shown as the graphs that better promote cooperation. In this article we have shown that the power-law degree distribution cannot be considered as the only root for the promotion of cooperation. At variance with the Barabási-Albert networks, the SF graphs considered in this work are free of any kind of node-node correlation. The first conclusion of our study is that cooperation decays when no correlations are present in the network. Moreover, we have shown that the organization of cooperation is dramatically different from that of the BA network, showing that cooperators can arrange in more than one cluster increasing the probability of being invaded by defectors. In other words, the fixation of cooperation is much lower than in SF networks with correlations, thus completing the picture provided by other studies where correlations were added into SF networks [Assenza, Gómez-Gardeñes \& Latora, 2008; Push, Weber \& Porto, 2008] enhancing the promotion of cooperation of BA networks. Therefore, one one hand, our study in random SF networks can be considered as the null model for the study of the cooperation in other types of SF graphs. On the other hand, our results highlight the importance of taking into account other structural properties beyond the degree distribution of the network [Da Fontura Costa, Rodrigues, Travieso \& Villas Boas, 2007] in order to capture the mechanisms that help to fixate cooperation in real complex networks.

The second part of the article presents a degree-based mean field approach to study analitycally networks with arbitrary degree distribution and no degree-degree correlations (such as random SF networks). The approach relies in a degree compartmentalization of cooperators and defectors strategists. We have shown that, contrary to diffusion dynamics where a similar approach has been applied, the degree-based mean field does not work correctly when general initial conditions are applied since no cooperation is observed when the temptation to defect is larger than the reward to cooperation. On the other hand, when a particular set of initial conditions is used (consisting in placing all the cooperators in the higher degree classes of the network) the solution of the mean field yields a non zero level of cooperation for a number of targeted initial configurations. The results obtained in this latter context qualitatively agree with those obtained when extensive numerical simulations on top of random SF graphs are performed.

As a conclusion, the results presented in this article complete the studies about the prisoner's dilemma on top of SF networks showing that node-node correlations play a key role for sustaining a high level of cooperation. Besides, the mean field approach, although not working for all the initial configurations placed in the networks, adds an analytical insight to the field of evolutionary games on graphs, being especially useful when targeted cooperation is studied. The mean field approach presented here is open to the incorporation of other ingredients such as degree-degree correlations that may help to reproduce the levels of cooperation observed in the numerical simulations.

\section{Acknowledgments}

This work has been partially supported by the DGA and the Spanish DGICYT Projects FIS2006-12781-C02-01 and FIS2005-00337. J.G.-G. and Y.M. are supported by the MICINN through the "Juan de la Cierva" and the "Ramón y Cajal" programs.

\section{REFERENCES}

Assenza, S., Gómez-Gardeñes, J., \& Latora, V. [2008], "Enhancement of cooperation in highly clustered scale-free networks", Phys. Rev. E 78, 017101.

Barabási, A.L., \& Albert, R. [1999], "Emergence of scaling in random networks", Science 286, 509-512.

Boccaletti, S., Latora, V., Moreno, Y., Chavez, M., \& Hwang, D.-U. [2006] "Complex networks: Structure and dynamics”, Phys. Rep. 424, 175-308. 
Bornholdt, S. \& Schuster, H. G. [2003] (Editors) "Handbook of Graphs and Networks" (Wiley-VCH, Germany).

Da Fontura Costa, L., Rodrigues, F. A., Travieso, G., \& Villas Boas, P. R. [2007], "Characterization of complex networks: A survey of measurements", Advances in Physics 56, 167-242.

Dorogovtsev, S. N. \& Mendes, J. F. F. [2003] "Evolution of Networks. From Biological Nets to the Internet and the WWW" (Oxford University Press, Oxford, U.K.).

Eguíluz, V.M., Zimmermann, M.G., Cela-Conde, C.J., \& San Miguel, M. [2005], "Cooperation and the emergence of role differentiation in the dynamics of social networks", Am. J. Soc. 110, 977-1008.

Floría, L.M, Gracia-Lázaro, C., Gómez-Gardeñes, J. \& Moreno, Y. [2008], "Social Network Reciprocity as a Phase Transition in Evolutionary Cooperation", e-print arXiv:0806.4962.

Gintis, H. [2000], "Game Theory Evolving” (Princeton University Press, Princeton, NJ).

Gómez-Gardeñes, J., Campillo, M., Floría, L.M., \& Moreno, Y. [2007], "Dynamical organization of cooperation in complex networks”, Phys. Rev. Lett. 98, 108103.

Gómez-Gardeñes, J., Poncela, J., Floría, L.M., \& Moreno, Y. [2008], "Natural selection of cooperation and degree hierarchy in heterogeneous populations", J. Theor. Biol. 253, 296-301.

Hamilton, W.D. [1964], “The genetical evolution of social behaviour", J. Theor. Biol. 7, 1-16.

Hofbauer, J., \& Sigmund, K. [1998], "Evolutionary Games and Population Dynamics", (Cambrige University Press, Cambridge, UK).

Hofbauer, J., \& Sigmund, K. [2003], "The evolution of cooperation", Bull. Am. Math. Soc. 40, 479-519.

Lieberman, E., Hauert, C., \& Nowak, M.A. [2005], "Evolutionary dynamics on graphs", Nature 433, 312-316.

Maslov, S., \& Sneppen, K. [2002], "Specificity and Stability in Topology of Protein Networks" Science 296, 910-913.

Molloy, M., \& Reed, B. [1998], "The Size of the Largest Component of a Random Graph on a Fixed Degree Sequence", Combinatorics, Probability and Computing 7, 295306.

Moreno, Y., Pastor-Satorras, R., \& Vespignani, A. [2002], "Epidemic outbreaks in complex heterogeneous networks",
Eur. Phys. J. B 26 521-529.

Newman, M.E.J. [2003], "The structure and function of networks", SIAM Rev. 45, 167-256.

Nowak, M.A. [2006], "Evolutionary Dynamics. Exploring the Equations of Life", (Harvard University Press, Harvard, MA).

Nowak, M.A., \& May, R.M. [1992], "Evolutionary games and spatial chaos", Nature 359, 826-829.

Nowak, M.A., \& Sigmund, K. [2005], "The evolution of cooperation" Nature 437, 1291-1298.

Ohtsuki, H., Hauert, C., Lieberman, E., \& Nowak, M.A. [2006], "A simple rule for the evolution of cooperation on graphs and social networks", Nature 441, 502-505.

Pastor-Satorras, R., \& Vespignani, A. [2001a], "Epidemic spreading in scale-free networks", Phys. Rev. Lett. 86, 32003203.

Pastor-Satorras, R., \& Vespignani, A. [2001b], "Epidemic dynamics and endemic states in complex networks", Phys. Rev. E 63, 066117.

Poncela, J., Gómez-Gardeñes, J., Floría, L.M., \& Moreno, Y. [2007], "Robustness of cooperation in the prisoner's dilemma in complex networks", New J. Phys. 9, 184.

Push, A., Weber, S., \& Porto, M. [2008], "Impact of Topology on the Dynamical Organization of Cooperation in the Prisoner's Dilemma Game" Phys. Rev. E 77036120.

Santos, F.C., \& Pacheco, J.M. [2005], "Scale-Free networks provide a unifying framework for the emergence of cooperation", Phys. Rev. Lett. 95, 098104.

Santos, F.C., \& Pacheco, J.M. [2006], "A new route to the evolution of cooperation", J. Evol. Biol. 19, 726-733.

Santos, F.C., Pacheco, \& J.M., Lenaerts, T. [2006], "Evolutionary dynamics of social dilemmas in structured heterogeneous populations", Proc. Natl Acad. Sci. USA 103, 34903494.

Szabó, G., \& Fáth, G. [2007], "Evolutionary games on graphs", Phys. Rep. 446, 97-216.

Szolnoki, A., Perc, M., \& Danku, Z. [2008], "Towards effective payoffs in the prisoner's dilemma game on scale-free networks", Physica A 387, 2075.

Vukov, J., Szabó, G., \& Szolnoki, A. [2008], "Evolutionary Prisoner's Dilemma game on the Newman-Watts networks", Phys. Rev. E 77, 026109. 